\title{
New Atomic Model with Identical Electrons Position in the Orbital's and Modification of Chemical Bonds and MOT
}

\author{
Abu Mohammad Azmal Morshed* \\ Department of Chemistry, Bangladesh University of Textiles (BUTEX), Bangladesh
}

\begin{abstract}
The concept of atom has undergone numerous changes in the history of chemistry, most notably the realization that atoms are divisible and have internal structure Scientists have known about atoms long before they could produce images of them with powerful magnifying tools because atoms could not be seen, the early ideas about atoms were mostly founded in philosophical and religion-based reasoning. This first atomic theory explained a lot, but as science continued to advance, new information and new discoveries made it clear that the theory needed to be updated. One of the most remarkable features of atomic theory is that even today, after hundreds of years of research, no one has yet seen a single atom. Some of the very best microscopes have produced images of groups of atoms, but no actual picture of an atom yet exists. How, then, can scientists be so completely certain of the existence of atoms and of the models they have created for them? The answer is that models of the atom, like other scientific models, can be tested by experimentation. Those models that pass the test of experimentation survive, while those that do not are abandoned. The model of atoms that scientists use today has survived and been modified by untold numbers of experiments and will be subjected to other such tests in the future. Even there are so many question arise about the chemical bond, MOT, VBT, VSEPR theory etc. by the existing atomic model. In my new atomic model, I have presented the definite position of electron/electron pairs in the different orbital (energy shells) with the identical distance among all nearby electron pairs and the degree position of electrons/electron pairs with the Center Point of Atoms (nucleus) in atomic structure, also in the molecular orbital. My new model will solve the confusions about MOT, VBT, VSEPR theory thus will explain the proper reasons of chemical bonding and many other unsolved questions of chemical combination and bond dissociation.
\end{abstract}

\section{Keywords}

Atom, Structure, Orbital, Molecule, Model

\section{Introduction}

An atomic theory is a model developed to explain the properties and behaviors of atoms [1-4]. As with any scientific theory, an atomic theory is based on scientific evidence available at any given time and serves to suggest future lines of research about atoms [5-7]. A proponent of the ultimate particle concept was the philosopher Democritus (c.470-c. 380 B.C.), who named those particles atoms. "Near the end of the $18^{\text {th }}$ century, new ways to conduct experiments helped researchers build their scientific understanding of atoms. This lead to the first atomic theory, which stated things like, all matter is made up of atoms, elements are composed of only one type of atom, and atoms can combine with other atoms to make more complex substances [8-10].

One of these new discoveries came at the end of the $19^{\text {th }}$ century, with the discovery of a subatomic particle, called an electron. When electrons were first discovered, scientists knew they were part of the atom, but they didn't know where they belonged. At first the thought was that electrons floated throughout the atom, like pieces of fruit floating in pudding, as if the atoms were simply soft blobs of matter. But, through more experimentation, it was found that most of the matter within atoms is centralized. By the early $20^{\text {th }}$ century, this centralized matter came to be recognized as the nucleus of the atom.

The idea that matter is made up of discrete units is a very old one, appearing in many ancient cultures such as Greece and India. The word "atom" was coined by the philosophers Leucippus and his pupil Democritus [2,3]. However, these ideas were founded in philosophical and theological reasoning rather than evidence and experimentation. Because of this, they could not convince everybody, so atomism was

*Corresponding author: Abu Mohammad Azmal Morshed, Department of Chemistry, Bangladesh University of Textiles (BUTEX), Tejgaon, Dhaka-1208, Bangladesh, Tel: +88-01674643058

Accepted: September 23, 2020

Published online: September 25, 2020

Citation: Morshed AMA (2020) New Atomic Model with Identical Electrons Position in the Orbital's and Modification of Chemical Bonds and MOT. Ann Atoms Molecules 2(1):6-13 

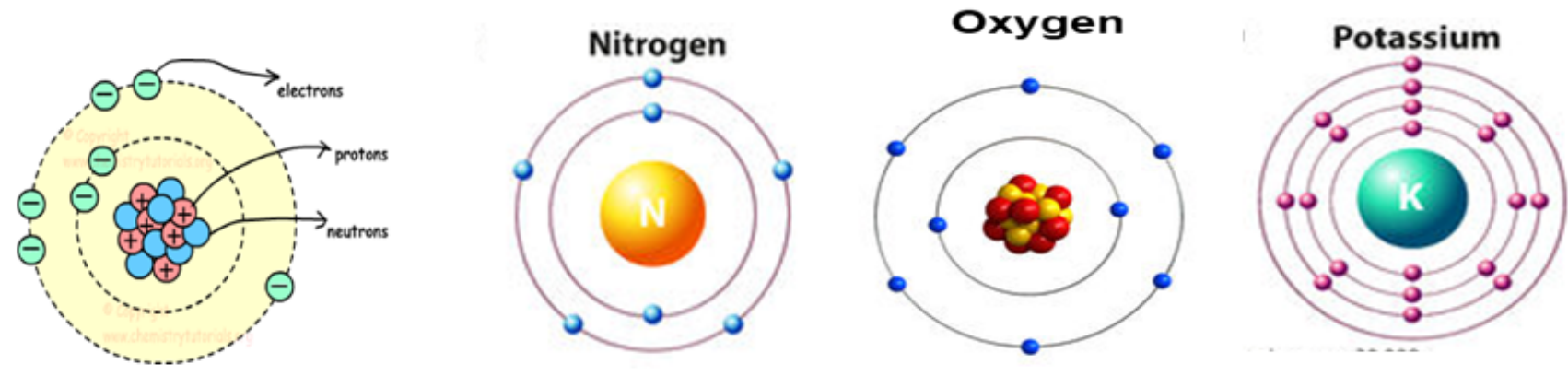

Figure 1: Existing atomic structure electrons arrangement around the nucleus where the electrons/electron pairs position is not identical by their position, alignments or distribution.

but one of a number of competing theories on the nature of matter. It was not until the $19^{\text {th }}$ century that the idea was embraced and refined by scientists, as the blossoming science of chemistry produced discoveries that could easily be explained using the concept of atoms [11-13].

John Dalton near the end of the $18^{\text {th }}$ century, two laws about chemical reactions emerged without referring to the notion of an atomic theory. The first was the law of conservation of mass, formulated by Antoine Lavoisier in 1789, which states that the total mass in a chemical reaction remains constant (that is, the reactants have the same mass as the products) [4]. The second was the law of definite proportions. First proven by the French chemist Joseph Louis Proust in 1799 [5] this law states that if a compound is broken down into its constituent elements, then the masses of the constituents will always have the same proportions, regardless of the quantity or source of the original substance.

Dalton proposed that each chemical element is composed of atoms of a single, unique type, and though they cannot be altered or destroyed by chemical means, they can combine to form more complex structures (chemical compounds). This marked the first truly scientific theory of the atom, since Dalton reached his conclusions by experimentation and examination of the results in an empirical fashion $[14,15]$.

\section{Aim and Scope of the Article}

Electrons are arranged in different orbital by different numbers in pairs/unpaired around the nuclei. Electrons pairs are associated by opposite spin together to restrict opposite movement for stability in orbital rather angular movements. The structural description is obeyed for the last more than hundred years but the exact positions of electrons/pairs in the energy shells of atomic orbital are not described with the exact locations among different orbital/shells. The existing electrons pair coupling model is not also shown clearly in figure by which a clear structure of opposite spine pair can be drowned. Also there are no proper distribution of electron/s around the center (nuclei) to maintain equal number of electrons/electronic charge (charge proportionality) around the total mass area of atomic circle $\left(360^{\circ}\right)$ in the existing atomic model (Figure 1). There are no clear ideas about the speed proportion and time of circulation of electrons/electron pairs in the atomic orbital/shells so there is no answer about the possibility of uneven number of electrons/electron pairs at any position /side of atomic body can arise that must make any atom unstable.

The existing atomic model/atomic structure cannot answer the reasons:

1. Why the similar charged electrons don't feel repulsion among themselves within the same nearby atomic orbital of same atom or even in the molecular orbital when two or more atomic orbital come closer to form molecular orbital within tinier space though there is more possibility of repulsion between similar charged electrons according to existing atomic model.

2. In case of ionic compound formation problem with the existing atomic model is where the transferred electron will take position in the new location on transferred atom? If the electrons position is not proportionally distributed along total 360 circulating area of atom, then the position of new transferred electron will cause the polarity in every ion (both cation and anion forms by every transformation of electrons) so the desired ionization is not possible thus every atom (ion) would become dipolar. On the point of view any ionization would not possible i.e., no ionic bonded compound would have formed.

3. The existing atomic model cannot explain the proper reasons of polarity of molecule.

4. The existing atomic structure/model cannot explain exact reason how dipole arise in the atomic body (dipolar atom) so the reason of Vandar Waals force, Dispersion force and London force is not clearly determined or understood yet.

5. In case of covalent bond (forms by sharing of electrons)

(i) There is no idea about the exact position of electrons/ opposite spine electron pairs in the atomic orbital.

(ii) There is no proper decision or explanation about the angle position of shared pair's electrons/electron pairs towards the nucleus in a covalent bond.

(iii) Why the similar charged electrons do not repulse each other in shared paired electron in molecular orbital and the existing atomic model cannot justify VSEPR theory.

(iv) How the attraction force (intermolecular force) acts/ 
arise within two nuclei of bonded atoms during molecule formation?

(v) How the bonded molecular orbital forms and how the attraction between opposite charged electrons (-ve) and proton (+ve) become greater force remitting the similar charged electrons (-ve)/electrons (-ve) and proton (+ve)/proton (+ve).

My new atomic model is designed on the basis of the position of electron(s)/electron pairs in the different energy shells including angular position towards the center i.e., nucleus in an/any atomic body which is able solve all the unsolved questions arise in the existing atomic model/structure.

\section{Discussion}

\section{New atomic model}

The new proposed atomic model presented in the article is based on the identical position of electrons/pairs around a $360^{\circ}$ diametertotal mass area of atoms. The atomic model is based on the exact position of electrons/pairs in the different orbital around nucleus in any atom. The model will also represent definite regular distance with all neighbor electrons/

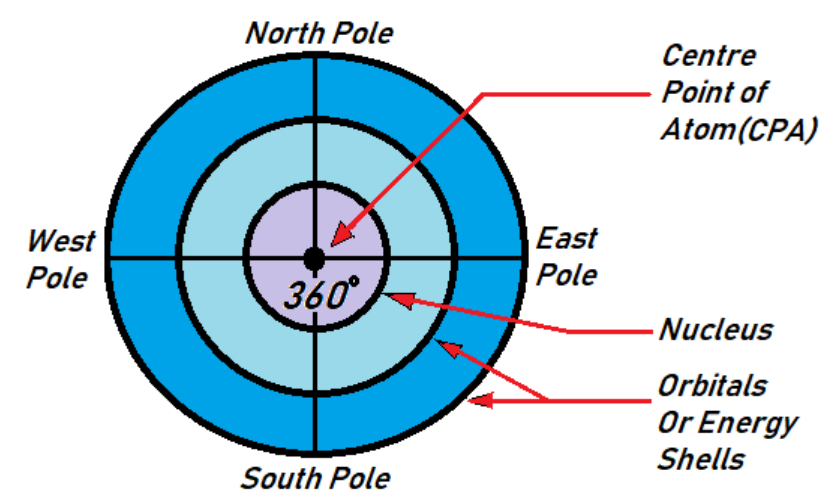

Figure 2: Total atomic area (360 ${ }^{\circ}$ total diameter) is divided into four poles (North Pole, South Pole, East Pole and West Pole). pairs along with angle position with the center of atomic nucleus within the atomic body.

\section{Atomic body is identified as four different poles}

For the better allocation of electron pairs position in the atomic orbital my new atomic model I have distributed first the total atomic area $\left(360^{\circ}\right.$ diameter mass area) into four poles (North Pole, South Pole, East Pole and West Pole) in (Figure 2).

\section{Center point of atom (CPA)}

Every atom contains a Center Point situated at the center of the atom (in nucleus), from the point atoms are $360^{\circ}$ spreaders around which form the round shape of atom including energy shells. The positive charges (proton) are arranged within or around the CPA.

\section{Center of electron pairs neutralization point (CENP)}

This is the pin-point situated in a middle position between two electrons of opposite spin pairs. The point is exactly between of opposite spine electron pairs so how the opposite electronic spin is neutralized to remaining a stable electron pair consisting of two opposite spin electrons. This CENP points are assumed to be situated between the cross section of opposite spine electronic pair's magnetic momentum field diameter (Figure 3).

CENP has a great role in the atomic association i.e., the similar charged (negative charged) electrons don't feel repulsion among themselves within the near most electron pairs or same orbital or/and different shells within the total atomic body. This mysterious point between opposite electron pair spin is the answer of the question why the near most similar charged electron pairs don't feel repulsion within the orbital in some molecular orbital in molecule. According to magnetic attraction/repulsion characteristics any similar charges repulse or opposite charges attract when the charges energy line is in straight points. If similar charged or opposite charged

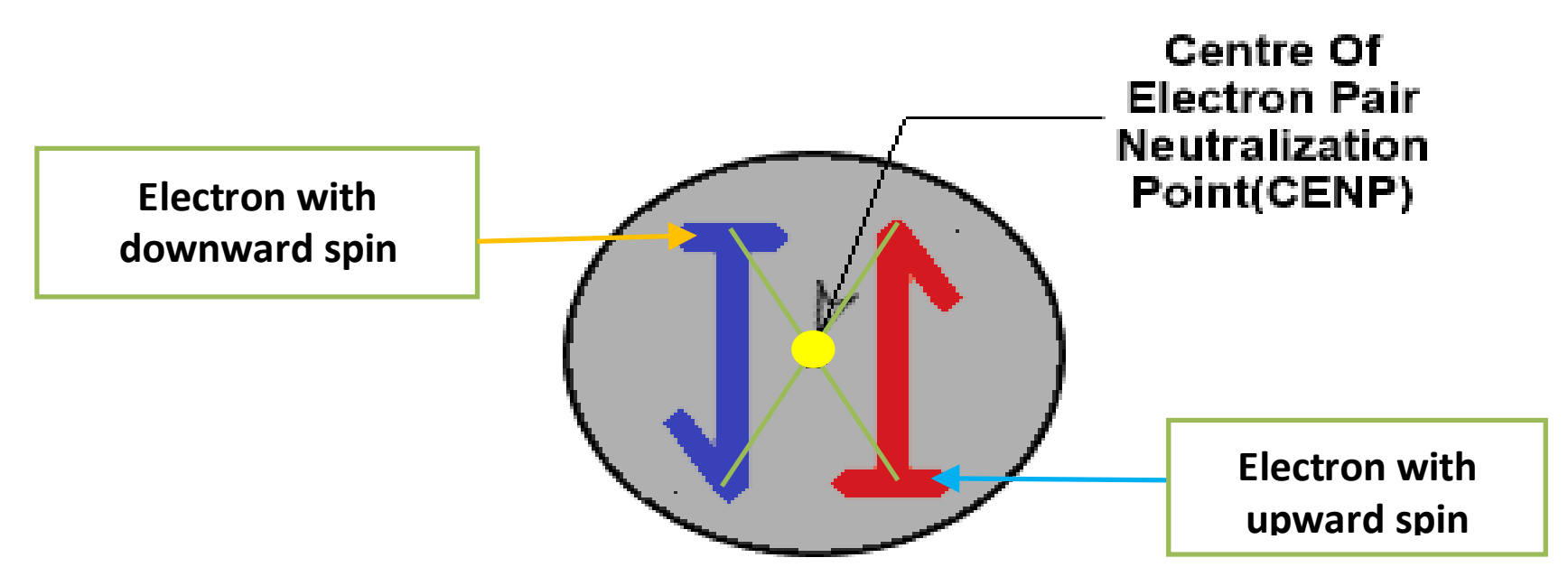

Figure 3: Showing an electron pair with Center of Electron Pair Neutralization Point (CENP) (a point which neutralize the opposite spin of electrons for stable and neutral electron pairs). 
end are even close but their center of energy points is not in straight line, there will be no attraction or repulsion between the charges (positive/negative). Similarly, when electrons are arranged in energy shells around the nucleus the electrons remain in pairs within opposite spin electrons where the poses a point which represent as the center of repulsion/attraction points (CENP) and two CENP never come to a straight within the atomic orbital so the similar charged electrons pairs don't feel repulsion within the energy shells. Another reason the CENP of all electron pairs are always straight to the Nucleus for the attraction between Positive Neutrons in Nucleus and negative charged electron pairs (Figure 3). Even with the changed position on the electron pairs around the Nucleus within the circulation atomic orbital the straight position between CENP and nucleus never effected i.e., the electron pairs revolves around nucleus maintaining the CENP straight to nucleus. This new atomic structure's Center of electron pairs neutralization points (CENP) concept gives the clear answer why the similar charged electrons don't feel repulsion force among themselves within same atomic orbital.

\section{Election's pair position in atomic orbital}

Total body of an atomic radius $\left(360^{\circ}\right)$ is divided into four parts across $90^{\circ}$ of cross section through the Center Point of the atom (CPA). The upper $90^{\circ}$ position with respect to CPA are named North pole ( $\mathrm{Np}$ ) and lower $90^{\circ}$ position is pointed as South pole (Sp) of the atom similarly right $90^{\circ}$ position with respect to CPA are named East Pole (Ep) and opposite West $90^{\circ}$ position are mentioned as West Pole $(\mathrm{Wp})$ in figure.

According to my new atomic model the angular position of electron pairs in different orbital is distributed keeping angular distance from each pair to another with respect to the center point of atom (mid-point of $360^{\circ}$ atomic body).

The angular distance calculations between different electron pairs among the various Energy Shells (Orbital) of same atoms are calculated using the following rules and the Mathematical Equation (1) (by AzmalMorshed) as follows:

Position of Electron(s)/electron pair(s) $=\mid\left\{\left(4 \pi r^{2} \div 4\right) \div\right.$ Number of Atomic Orbital $\} \div$ Orbital position number counted from outside orbital\}].

(Starting from inner most energy shell to outer most energy shells)

\section{Modification of ionic bond concept according to new atomic model}

Ionic bonds are formed by the electron transformation between bonded atoms, Metallic atom donate electrons to the to the nonmetallic partner then the metallic atom gets positively charged ion by losing negative charge result excess proton (positive charge) in nucleus. Similarly, the nonmetallic atom gets negatively charged ion due to the excess electron (negative charge) results excess difference in electron number (negative charges) in energy shells with proton number (positive charge) in nucleus. Then the opposite charged ion results the ionic bond by reducing inter nuclear distance. Now question arise the about the position of transferred electron:

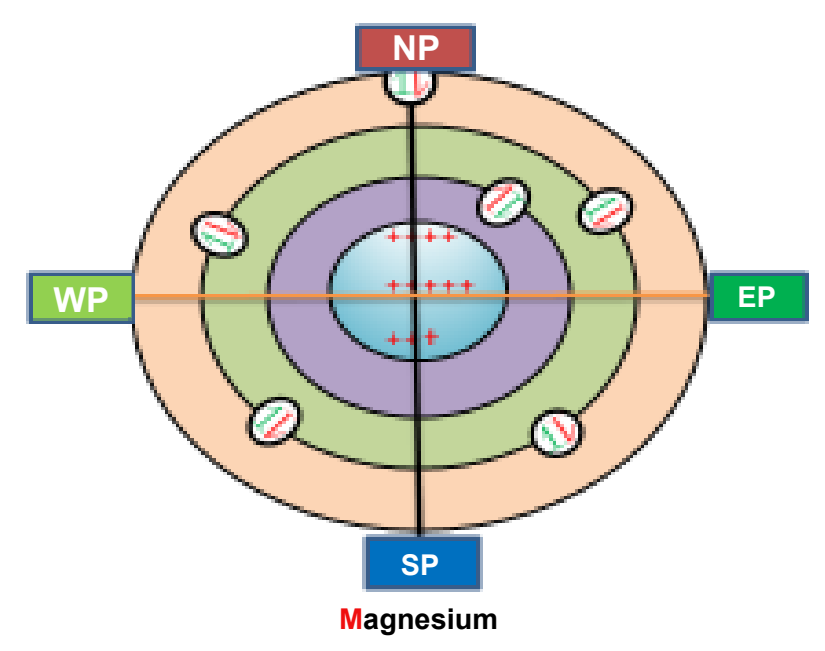

Figure 4: Atomic structure of Magnesium an example according to new arrangement of electron pairs arrange by proper degree angle with the CPA also showing the center of every electron pairs directed to the CPA for attraction and proving the impossibility of repulsion between any two of neighbor electrons/pairs or even among any similar charged pair electrons.

(a) From which position the electron/s go to which position of the new transferred orbital? If the transferred electron/s doesn't make a balanced condition around $360^{\circ}$ atomic mass body during or after the ion formation, then every ion would a polar ion in fact. The existing atomic model/ electronic configuration failed to answer the arise question about neutral ion formation and ionic bond. My new atomic model with identical electrons pair angle position is able to give logical solution to the problems of ion/ionic bond formation. As follows: The metallic atom which donate electrons during ion formation from outermost orbital, the electrons are arranged maintaining definite degree angle around $360^{\circ}$ atomic mass body shown in (Figure 4). After the transformation the transferred electron take position at the vacant place of the transferred atoms outermost orbital, then instant the near most electrons/pairs rearrange their position in the orbital changing their angle position with the CPA due to electromagnetic repulsion feeling among the similar charged electrons/pairs. Thus the ionic atom gets equal electron charge density around whole of their $360^{\circ}$ atomic mass body resulting the cation and anion due to the positive and negative charge difference in atomic orbital with their respective nucleus. Thus every ion becomes non polar ion to form ionic bond within two opposite charged ion (Figure 5).

\section{Modification of covalent bond concept accord- ing to new atomic model}

Covalent bonds are formed by sharing of electrons between the bonded atoms and the shared paired electrons are formed by contribution of one electron each of the participating atoms. The shared paired electrons remain at the overlapping chamber (at the juncture of the overlapped atomic orbital). The present structural presentation of any molecule it is show that the shared paired electrons remain at the over- 


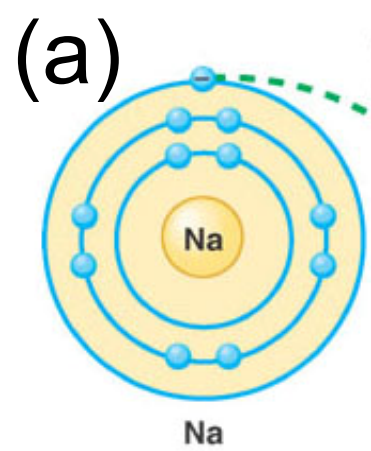

Sodium atom

\section{Transfer of} electron $\because$

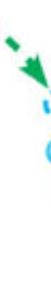

4

(b)

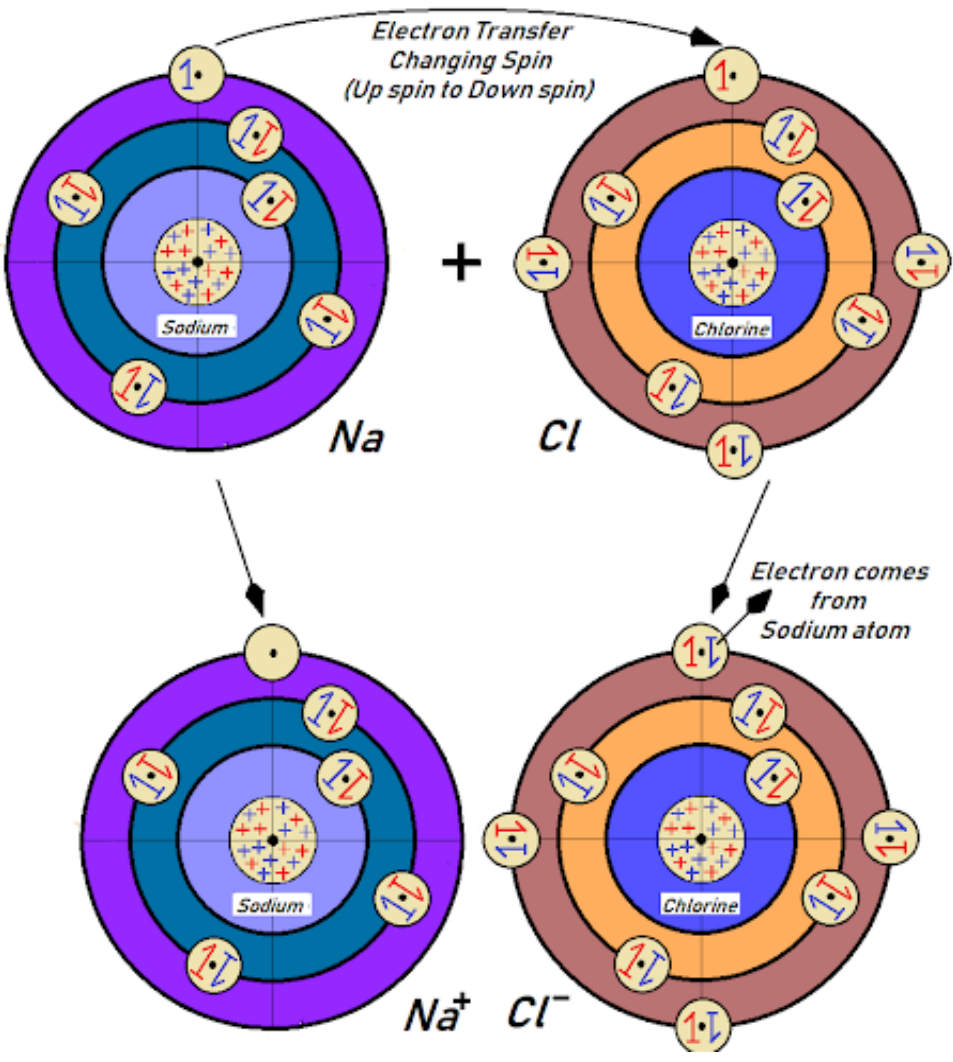

Figure 5: (a) Electron transfer for ion formation according to existing method; (b) Electron transfer for ion formation in ionic bond according to new proposed method.

lapping chamber but there is no presentation about their spin and exact position in the overlapping chamber. According to present structural presentation of shared paired electrons remain at the juncture of the bonded atomic orbital, if they remain like such position they will restrict the Inter Molecular Force (IMF) between the bonded atomic nuclei because the shared paired electron restricts the attraction force lying at the straight attraction line of the bonded nuclei the shown in (Figure 6a). According to the new covalent bond pictorial presentation in light of new atomic model; the shared paired electron making a proper pair by opposite spin along with a CENP and will take place at the joint point (overlapping chamber) in such a way, there will be no restriction on way of attraction (IMF)/repulsion between the bonded nuclei shown in (Figure 6b).

\section{New solution about MOT by the new atomic model}

Similar solution can be made for molecular orbital (MOT) as such as: The molecular orbital (MO) theory is described as a method for determining molecular structure in which electrons are not assigned to individual bonds between atoms, but are treated as moving under the influence of the nuclei in the whole molecule. Still it is believed that when the molecules formed by sharing electrons, the sharing electrons pair remain in the overlapping chamber (junction of the bonded atoms) shown in (Figure 7a). 


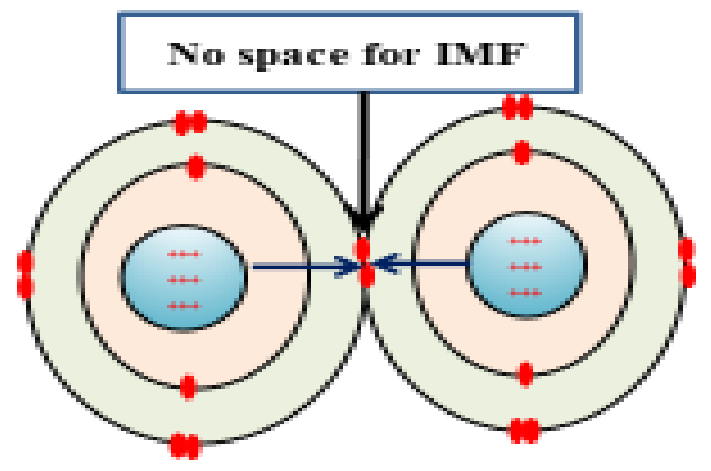

(a)Fluorine Molecule $F_{2}$

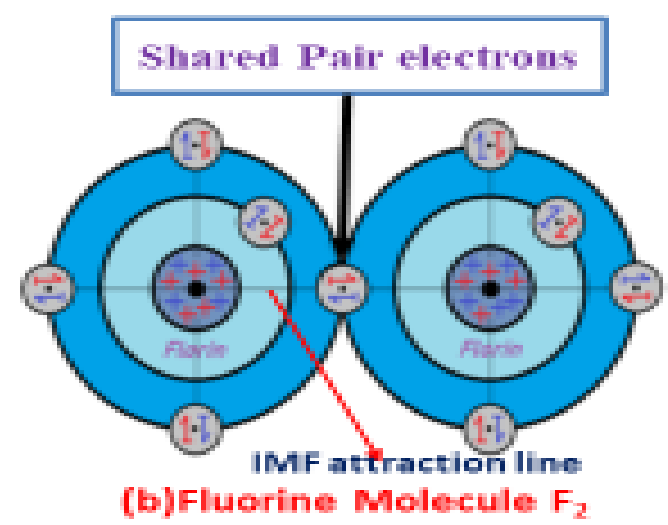

(b)Fluorine Molecule $F_{2}$

Figure 6: (a) Existing Covalent bond structural presentation of Fluorine molecule showing the shared paired electrons without proper opposite balanced spin structure and no space for IMF; (b) Showing the shared paired electrons with opposite spin and clear connection between to bonded atomic nuclei for IMF.

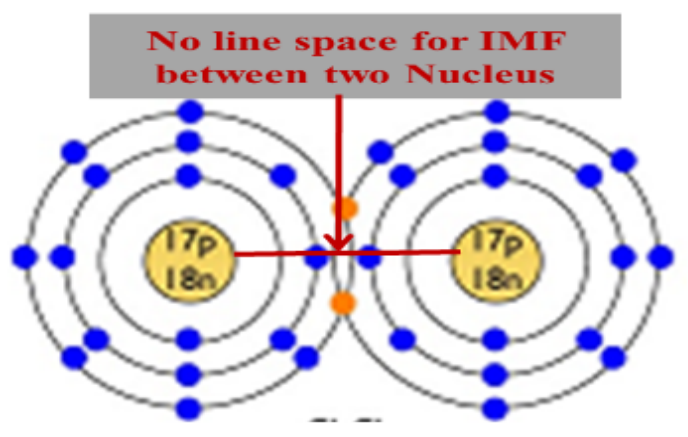

(a)Chlorine Molecule $\mathrm{Cl}_{2}$

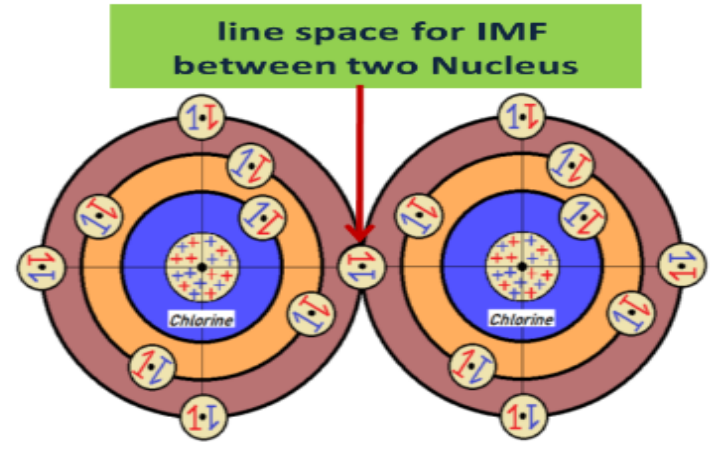

(b)Chlorine Molecule $\mathrm{Cl}_{2}$

Figure 7: (a) Existing structure of Chlorine Molecule: In the structure there is no space between two bonded nucleuses for the Intermolecular Force of Attraction (IMF) also the possibility of repulsion between two atomic orbital nearby electrons/pairs; (b) Designed according to new atomic model where there is a clear line space for IMF between two bonded atomic Nuclei. Also showing there is no possibility of repulsion among the nearby similar charged electrons/pairs because there are no two CENP in same line.

But main drawbacks of the existing MOT are:

(i) There is no clear concept about the position of the sharing electron pairsand their exact opposite spin structural position in the juncture of overlapped two atomic orbital in a molecular orbital, and the existing atomic model and MOT cannot explain the proper reason of attraction between the bonded atoms nuclei (inter molecular force of attraction) also "why there are no repulsion between near most two nuclei in a MO themselves?".

(ii) When two atomic orbital's combine to form a molecular orbital, the situation described by VSEPR theory is not satisfactory because it said when two atomic orbital come close to form a molecular orbital there remain repulsion between the opposite charged electrons/electron pairs up to certain extend and the similar repulsion situation turns into attraction forces when the atomic orbital come closer: is a funny description. According to my new atomic model the arrangement of electron pairs in different orbital of any atom are arranged such way that the opposite spine electron pair's attraction/repulsion point Center of
Electron Pair Neutralization Point(CENP) (Figure 2) will always remain towards nucleus of the respective atoms and never will in a line of other electrons/pairs CENP even never with the CENP of any electrons/pairs of nearby atomic orbital electron pairs during molecular orbital formation or even in normal beside staying atoms. So there are no possibilities of repulsion between the upcoming nearby electron pairs (described in VSEPR theory on the basis of existing atomic model) during molecular orbital formation (Figure 4) new atomic model is followed during the molecular orbital are formed. What about the repulsion between more nearest similar charged electrons (-Ve charged) in a molecular orbital? Most importantly how inter nuclear force of attraction i.e. Inter Molecular Force of Attraction (IMF) forms between two similar charged (+Ve charged) Nuclei rather than repulsion? The questions have no proper and logical answer to the world scientist with the existing atomic model because problem is in the position of electrons in atomic orbital and their direction towards nucleus. My new atomic model can answer the entire question about the Molecular Orbital formation. 
The shared pair electrons in the molecular orbital (in the overlapping chamber/junction of the bonded atoms) according to my new model of electron pair where they contain identical CENP (Center of Electron Pairs Neutralization Point). The CENP remain as straight to both nuclei of bonded atoms in molecules. So the CENP (Center of Electron Pairs Neutralization Point) works as the binding points of attraction between two nuclei in a molecular orbital providing facility of attraction force among both the nuclei. The CENP (Center of Electron Pairs Neutralization Point) in the shared electron pairs in a molecular orbital is responsible that the similar charged Nuclei don't feel repulsion force which turns into attraction forces because of the CENP position in the straight line between two CPA (Center Point of Atom) bonded atoms. Ultimately for the absence of electron-electron repulsions between the bonded atoms electron pairs in the same atoms or even with the near most atoms electron pairs in a molecular orbital attraction between bonded atoms electron pairs is the only force between two bonded nuclei is the intermolecular force of attraction (IMF).

According to my new model of atomic structure all the electron(s)/electron pairs CENP (Center of Electron Pairs Neutralization Points) are straight directed always to the nucleus of respected atoms from different angle position around the $\mathbf{4} \pi \mathbf{r}^{2}$ total atomic radius. The electron pairs CENP (Center of Electron Pairs Neutralization Points) never come in between or straight to another electron pair CENP. There is no possibility of repulsion between similar charged electron(s)/pair(s) in an atomic orbital (Figure 7b). Similarly, reason arise when two or more atomic orbital come closer to each other for molecule formation any of their electron pairs CENP (Center of Electron Pairs Neutralization Points) never become straight to one another in the molecular orbital (MO) formation.

\section{Modification in double bonded molecular struc- ture}

It is shown for an Oxygen molecule; two electron shared pairs are formed and take place at the overlapping chamber result from the outermost orbital of two bonded Oxygen atoms. But in real séance that is impossible because two shared paired electrons cannot remain in a single overlapping chamber because of repulsion among each pairs and among individual electrons. The existing molecule concept hasn't any explanation about how the shared paired electron forms with opposite spin pair with single electrons coming from both of the atomic orbital of Oxygen with a spin. The main problem with the present Oxygen molecular structure is that we know there are a double bond (double bond consist a sigma bond formed by side to side overlapping of the bonded atomic orbital and a pi-bond forms by the head to head overlapping of the bonded atomic orbital) exist between two Oxygen atoms in an Oxygen molecule. The present structure of $\mathrm{O}_{2}$ molecule show only two pairs of electron with head to head overlapping in the overlapping chamber i.e., two sigma bond together which is impossible (Figure 8a). According to my new given model for Oxygen molecule I have shown that the only a shared paired electrons take place at the overlapping chamber by the combination of opposite spin electrons coming from the different Oxygen atom (b) resulting a head to head overlapped sigma bond. The remaining two opposite spin unpaired electrons in the two bonded Oxygen's outermost orbital getting little distorted towards the shared paired electrons in their respective atomic orbital then they feel an attraction among the opposite spin electrons thus they make a bond pairs by side to side overlapping forms the pi-bond (Figure 8b). The intermolecular force of attraction is (IMF) is clearly maintained by a straight line attraction line through the shared electron pair's CENP according to the new molecular structural presentation. Similar modification is required for few other double bonded molecules $\left(\mathrm{Eg} . \mathrm{CO}_{2}\right.$ ) following the new concept of molecule structure.

\section{Conclusion}

The new atomic model will assist the world to open new windows of knowledge of understanding about different types of chemical bonding, bond strength, electrons charge density (electrons cloud), exact reasons of polarity in compounds, molecule and atoms. The concept is able to explain the reasons of different reactivity of atoms, molecules and
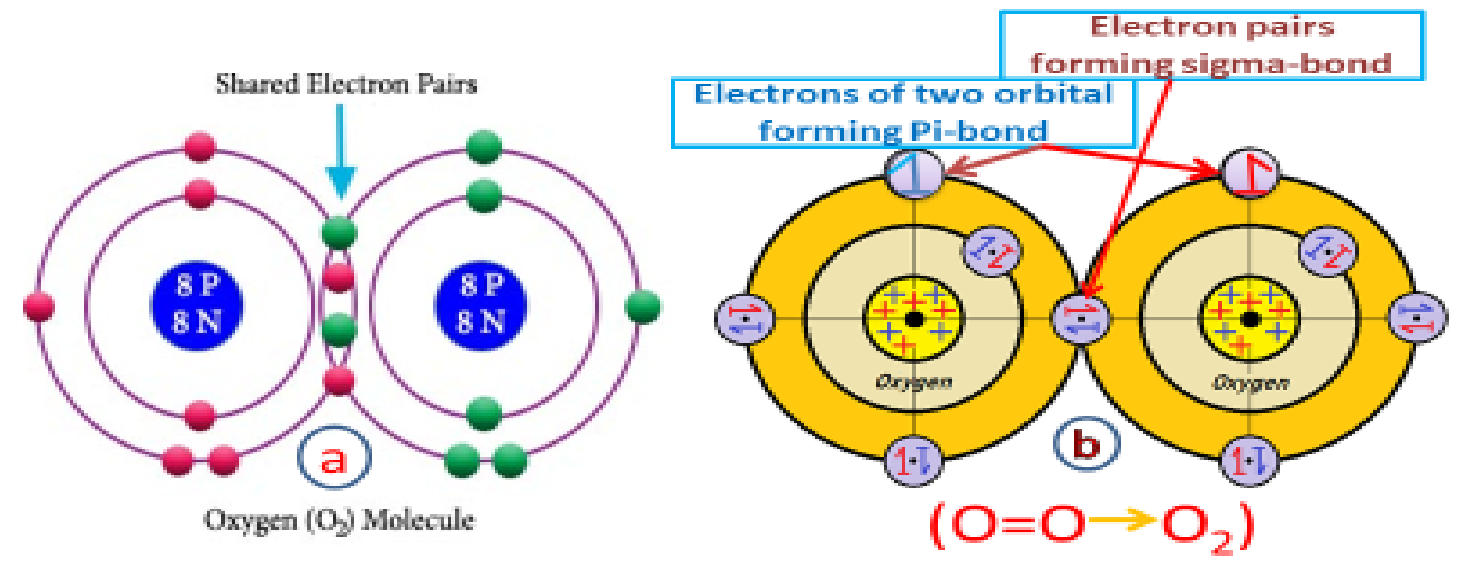

Figure 8: (a) Present molecular orbital structural showing two shared paired electrons in the same overlapped chamber with head to head overlapping results two sigma bonds; (b) New molecular orbital structure clearly showing two different Sigma and pi-bond proves the existing of double bond in Oxygen molecule also showing clear IMF line between two bonded Oxygen nucleus. 
compounds. The new concept is also capable to answer many unresolved problems in the atomic charge concept, ionization potential, causes of unusual properties in chemical bodings and can elucidate the properties of compounds. I am hopeful that the new atomic model will introduce new horizon in the quantum mechanics acquaintance.

\section{Acknowledgement}

I am highly thanks to my family members and colleagues of Bangladesh University of Textiles (BUTEX) for their endless encouragement. I would like to pay my gratitude to the respected Dean, Faculty of Science and Engineering and to our honorable Vice Chancellor.

\section{Author Contribution}

As an author of the article I am declaring that the total works is originally carried me only. There is no conflict with any other's work I know and it was not borrowed or copied from any other works.

\section{References}

1. Pullman Bernard (1998) The atom in the history of human thought. Oxford University Press, Oxford, England, 31-33.

2. Cohen Henri, Lefebvre Claire (2017) Handbook of categorization in cognitive science. $\left(2^{\text {nd }}\right.$ edn), Elsevier, Amsterdam, The Netherlands, 427.

3. Henry M Leicester, Herbert S Klickstein (1952) A source book in chemistry. 1400-1900.

4. Dalton John (1806) On the absorption of gases by water and other liquids. Memoirs of the Literary and Philosophical Society of Manchester 24: 15-24.
5. Johnson Chris (2002) Avogadro-his contribution to chemistry. Archived from the original 07-10.

6. Avogadro Amedeo (1811) Essay on a manner of determining the relative masses of the elementary molecules of bodies, and the proportions in which they enter into these compounds. Journal de Physique 73: 58-76.

7. Thomson JJ (1897) Cathode rays. Philosophical Magazine 269: 293-316.

8. Thomson JJ (1904) On the structure of the atom: An investigation of the stability and periods of oscillation of a number of corpuscles arranged at equal intervals around the circumference of a circle; with application of the results to the theory of atomic structure. Philosophical Magazine 7: 237-265.

9. Rutherford Ernest (2011) The scattering of $\alpha$ and $\beta$ particles by matter and the structure of the atom. Philosophical Magazine 92: 379-398.

10. Bohr Niels (1913) On the constitution of atoms and molecules. Philosophical Magazine 26: 476-502.

11. Thomson JJ (1913) Rays of positive electricity. Proceedings of the Royal Society 89: 1-20.

12. Rutherford Ernest (1919) Collisions of alpha particles with light atoms. IV. an anomalous effect in nitrogen. Philosophical Magazine 37: 581-587.

13. Chadwick James (1932) Possible existence of a neutron. Nature 312.

14. Schrödinger Erwin (1926) Quantisation as an eigenvalue problem. Annalen der Physik 18: 109-139.

15. Heisenberg W (1927) Über den anschaulichenInhalt der quantentheoretischenKinematik und Mechanik. ZeitschriftfürPhysik 43: 172-198. 